\title{
Improved calculation of the equilibrium magnetization of arterial blood in arterial spin labeling
}

\author{
Ahlgren, Andre; Wirestam, Ronnie; Knutsson, Linda; Petersen, Esben Thade
}

Published in:

Magnetic Resonance in Medicine

Link to article, DOI:

$10.1002 / \mathrm{mrm} .27193$

Publication date:

2018

Document Version

Peer reviewed version

Link back to DTU Orbit

Citation (APA):

Ahlgren, A., Wirestam, R., Knutsson, L., \& Petersen, E. T. (2018). Improved calculation of the equilibrium magnetization of arterial blood in arterial spin labeling. Magnetic Resonance in Medicine, 80(5), 2223-2231. https://doi.org/10.1002/mrm.27193

\section{General rights}

Copyright and moral rights for the publications made accessible in the public portal are retained by the authors and/or other copyright owners and it is a condition of accessing publications that users recognise and abide by the legal requirements associated with these rights.

- Users may download and print one copy of any publication from the public portal for the purpose of private study or research.

- You may not further distribute the material or use it for any profit-making activity or commercial gain

- You may freely distribute the URL identifying the publication in the public portal 


\section{Improved calculation of the equilibrium magnetization of arterial blood in arterial spin labeling}

André Ahlgren (1), Ronnie Wirestam (1), Linda Knutsson (1,2), Esben Thade Petersen (3,4)

(1) Department of Medical Radiation Physics, Lund University, Lund, Sweden

(2) Department of Radiology, Johns Hopkins School of Medicine, Baltimore, US

(3) Danish Research Centre for Magnetic Resonance, Centre for Functional and Diagnostic Imaging and Research, Copenhagen University Hospital Hvidovre, Hvidovre, Denmark

(4) Center for Magnetic Resonance, DTU Elektro, Technical University of Denmark, Kgs Lyngby, Denmark

\section{Corresponding author:}

André Ahlgren

E-mail address: andreahlgren@gmail.com

Telephone: +46 46-173146

Fax: +46 46-178540

Address:

Department of Medical Radiation Physics

Lund University

Barngatan 4

Skåne University Hospital, Lund

SE-221 85 LUND

Sweden

Word count: 3364

Number of figures: 5

Number of tables: 0

Number of references: 28

Manuscript type: Note

Keywords: arterial spin labeling, perfusion, cerebral blood flow, equilibrium magnetization of arterial blood, partition coefficient, fractional signal modeling, partial volume

Running title: Improved calculation of $M_{0 a}$ in arterial spin labeling 


\section{Abstract}

\section{Purpose}

To propose and assess an improved method for calculating the equilibrium magnetization of arterial blood $\left(M_{0 a}\right)$, used for calibration of perfusion estimates in arterial spin labeling (ASL).

\section{Methods}

Whereas standard $M_{0 a}$ calculation is based on dividing a proton density weighted image by an average brain-blood partition coefficient, the proposed method exploits partial volume (PV) data to adjust this ratio. The nominator is redefined as the magnetization of perfused tissue, and the denominator is redefined as a weighted sum of tissue-specific partition coefficients. Perfusion data were acquired with a pseudocontinuous ASL sequence, and PV data were acquired using a rapid saturation recovery sequence with the same readout module. Results from 7 healthy volunteers were analyzed and compared with the conventional method.

\section{Results}

The proposed method produced improved $M_{0 a}$ homogeneity throughout the brain in all subjects. The mean gray matter perfusion was significantly higher with the proposed method compared to the conventional method; 61.2 versus $56.3 \mathrm{ml} / 100 \mathrm{~g} / \mathrm{min}(+8.7 \%)$. Although to a lesser degree, the corresponding white matter values were also significantly different; 20.8 versus $22.0 \mathrm{ml} / 100 \mathrm{~g} / \mathrm{min}(-5.4 \%)$. The spatial and quantitative differences between the two methods were similar in all subjects.

\section{Conclusion}

Compared to the conventional approach, the proposed method produced more homogenous $M_{0 a}$ maps, corresponding to a more accurate calibration. The proposed method also yielded significantly different perfusion values across the whole brain, and performed consistently in all subjects. The new $M_{0 a}$ method improves quantitative perfusion estimation with ASL, and can therefore be of considerable value in perfusion imaging applications. 


\section{Introduction}

Arterial spin labeling (ASL) is a well-established non-invasive perfusion MRI technique, increasingly used in clinical applications [1]. Ever since the original ASL work by Detre et al. and Williams et al. [2,3], the equilibrium magnetization of arterial blood water $\left(M_{0 a}\right)$ has been identified as the central calibration factor for converting perfusion weighted signal to quantitative perfusion values. An accurate $M_{0 a}$ calculation is thus essential to obtain accurate perfusion estimates. The $M_{0 a}$ factor represents the signal from a voxel completely filled with arterial blood, and the value therefore relates to the theoretical maximum of the ASL difference signal.

The most common approach to estimate $M_{0 a}$ is by dividing the equilibrium voxel magnetization $\left(M_{0}\right)$ by the brain-blood partition coefficient for water $(\lambda)$. A separately acquired proton density (PD) weighted image is often used for $M_{0}$, although control data from the ASL scan can also been used (if the ASL sequence does not utilize background suppression). For $\lambda$, an average whole-brain partition coefficient based on literature values is usually employed [3].

Since the theoretical calibration factor is global, other approaches based on reference signals have also been used for calculation of $M_{0 a}$. For example, the $M_{0}$ value in white matter (WM), cerebrospinal fluid (CSF) or sagittal sinus can be used in combination with appropriate corrections for water content, relaxation times and brain density [4-7]. The advantage of calculating voxel-wise $M_{0 a}$ values, however, is that B1- field inhomogeneities and $\mathrm{T} 2^{(*)}$ contrast are automatically corrected for in the perfusion calculation [7,8].

A drawback of the conventional approach is that the $M_{0 a}$ map will be PD-weighted, leading to bias (inverse PD-weighting) in the perfusion maps, mainly because differences in $\lambda$ between gray matter (GM) and WM are not accounted for $[9,10]$. Furthermore, since the conventional $M_{0 a}$ calculation does not account for the fact that cerebrospinal fluid (CSF) is non-perfused, bias can also arise due to partial volume of CSF in the voxel (e.g., in cortical GM). In other words, $\lambda$ is proportional to the voxel-wise tracer distribution volume [11], which is seldom taken into account in ASL.

In this work, we propose an improved method for calculation of $M_{0 a}$, based on partial volume (PV) mapping. The analysis of saturation recovery (SR) data allows for voxel-wise estimation of the equilibrium magnetization of perfused tissue $M_{0 t}$, and a tissue-weighted partition coefficient $\lambda_{w}$, used to calculate an accurate $M_{0 a}$ map. Using in vivo data, the novel method is compared with the conventional approach in terms of visual maps, quantitative differences, and statistical differences.

\section{Methods}

\section{Theory}

The partition coefficient of a tracer is the ratio between the tissue and blood tracer concentrations at equilibrium, and it is thus proportional to the tracer distribution volume [9,11]. It is used to correct tracer kinetic parameters for the corresponding difference in signal between blood and tissue. In ASL, labeled blood water is the tracer, and tracer concentration is therefore represented by water density. Since magnetization is proportional to water density, the ratio of water density in tissue $\left(\rho_{t}\right)$ to blood $\left(\rho_{a}\right)$ can be written

$$
\frac{\rho_{t}}{\rho_{a}}=\frac{M_{0 t}}{M_{0 a}}=\lambda
$$


where $M_{0 t}$ and $M_{0 a}$ are the equilibrium magnetizations in tissue and blood, respectively, and $\lambda$ is the brainblood partition coefficient for water in units (quantity of water $/ \mathrm{ml}$ tissue $) /($ quantity of water $/ \mathrm{ml}$ blood $)=(\mathrm{ml}$ blood $) /(\mathrm{ml}$ tissue) [3,9]. It is common to express $\lambda$ in units of $(\mathrm{ml} \mathrm{blood}) /(\mathrm{g}$ tissue $)$, so that $\lambda$ represents the volume of distribution of water per unit mass of tissue. Traditionally, literature values based on nuclear medicine measurements are used for $\lambda$ [9]. Although some tissue water may be MR invisible, so that $\lambda$ in ASL could differ from that in nuclear medicine [12], MRI-based measurements are in good agreement with the established values [13].

The conventional $M_{0 a}$ calculation in ASL corresponds to

$$
M_{0 a}^{c}=\frac{M_{0}}{\bar{\lambda}}
$$

where $\bar{\lambda}$ is the whole-brain average partition coefficient. This conventional calculation is not strictly correct, since it does not accurately account for the voxel-wise distribution volume of blood water.

Acknowledging that the labeled blood water does not distribute in CSF, the equilibrium magnetization of perfused parenchymal tissue is given by

$$
M_{0 t}=M_{0}\left(1-m_{C S F}\right)
$$

where $m_{C S F}$ is the fractional magnetization of CSF. A tissue-weighted brain-blood partition coefficient can be calculated according to

$$
\lambda_{w}=w_{G M} \lambda_{G M}+w_{W M} \lambda_{W M}
$$

where $w_{G M}$ and $w_{W M}$ are the fractional weights, and $\lambda_{G M}$ and $\lambda_{W M}$ are the partition coefficients, of GM and WM, respectively [11]. Finally, the proposed $M_{0 a}$ calculation with appropriate adjustment for the distribution volume of labeled blood water is given by

$$
M_{0 a}=\frac{M_{0 t}}{\lambda_{w}}=\frac{M_{0}\left(1-m_{C S F}\right)}{w_{G M} \lambda_{G M}+w_{W M} \lambda_{W M}}
$$

\section{Data acquisition}

MRI data acquisition was performed at 3T (Achieva, Philips Healthcare, Best, The Netherlands) using a 8channel head coil. Seven healthy subjects (4 M, mean age 33 y) gave written informed consent, and the study was approved by the local ethics committee. Pseudo-continuous ASL (PCASL) data were acquired using label duration $=1650 \mathrm{~ms}$, post-label delay $=1550 \mathrm{~ms}$, background suppression, vascular flow crushing, averages $=29$, repetition time $=4 \mathrm{~s}$, at a scan time of $3 \mathrm{~min} 52 \mathrm{~s}$. The labeling module employed Hanning pulses of $0.5 \mathrm{~ms}$ duration, $1 \mathrm{~ms}$ pulse interval, $18^{\circ}$ flip angle and the mean gradient strength was $0.6 \mathrm{mT} / \mathrm{m}$. The PV data were acquired using a SR sequence with 'water suppression enhanced through T1 effects' (WET) saturation, shortest repetition time, nine saturation times $(0 / 250 / 500 / 750 / 1000 / 1500 / 2000 / 3000 / 4000 \mathrm{~ms})$, at a scan time of $18 \mathrm{~s}$. Both sequences used an identical multi-slice single-shot echo-planar imaging (EPI) readout module with $3.75 \times 3.75 \times 8 \mathrm{~mm}^{3}$ voxel size, 13 slices, $13 \mathrm{~ms}$ echo time, $33 \mathrm{~ms}$ slice time readout, fat saturation, and a SENSE factor of 2.5. To avoid issues with different scanner gain in the SR reference and ASL acquisitions, the same preparation phases were used for all scans. 


\section{Post-processing}

The $M_{0}$ map, as well as fractional magnetizations, weights and volumes $\left(p_{i}\right)$, were acquired from the SR data using the fractional signal modeling (FSM) approach [14]. In short, the SR signal equation $S(t)=$ $M_{0}\left(1-e^{-t / T_{1}}\right)$ was nonlinearly fit to the SR data with $M_{0}$ and $T_{1}$ as free parameters. Thereafter, a multiGaussian model (4 peaks corresponding to GM, WM, GM/CSF and GM/WM) was nonlinearly fit to a whole-brain $R_{1}=1 / T_{1}$ histogram. Fitting to $R_{1}$ rather than $T_{1}$ was empirically found to be more robust to initial fit parameter values. The mean values for the GM and WM peaks were used as representative $T_{1}$ values for those tissue types, and the $T_{1}$ in CSF was set to $4.3 \mathrm{~s}$, based on literature values [14]. The signal fractions were then estimated by linear fitting of the SR-based FSM model given by

$$
S(t)=\sum_{i} s_{i}\left(1-e^{-t / T_{1 i}}\right)
$$

where $s_{i}$ is the equilibrium magnetization of compartment $i$, and $T_{1 i}$ is the longitudinal relaxation time of compartment $i$. Fractional magnetizations, weights and volumes were obtained by appropriately scaling with water contents, densities, and normalization, i.e., $m_{i}=s_{i} / \sum_{i^{\prime}} s_{i^{\prime}}$, $p_{i}=\left(s_{i} / \rho_{i}\right) / \sum_{i^{\prime}} s_{i^{\prime}} / \rho_{i^{\prime}}$, and $w_{i}=\left(s_{i} \varrho_{i} / \rho_{i}\right) / \sum_{i^{\prime}} s_{i^{\prime}} \varrho_{i^{\prime}} / \rho_{i^{\prime}}$, where $\rho_{i}$ is the water density of compartment $i$ (set to $1.00 / 0.89 / 0.73$ for CSF/GM/WM [14]) and $\varrho_{i}$ is the mass density of compartment $i$ (set to $1.00 / 1.04 / 1.04 \mathrm{~g} / \mathrm{ml}$ for CSF/GM/WM).

The proposed and conventional $M_{0 a}$ estimates were calculated according to Eqs. 4 and 5, using literature values for partition coefficients, i.e., $\lambda_{G M}=0.98 \mathrm{ml} / \mathrm{g}, \lambda_{W M}=0.82 \mathrm{ml} / \mathrm{g}$ and $\bar{\lambda}=0.9 \mathrm{ml} / \mathrm{g}$ [9]. The $M_{0}$ from the SR fit was used in both the proposed and the conventional approach. The perfusion was quantified using the basic PCASL model given by

$$
\Delta M=2 M_{0 a} \alpha f T_{1 a}\left(1-e^{-\tau / T_{1 a}}\right) e^{-w / T_{1 a}}
$$

where $\Delta M$ is the ASL signal difference, $\alpha$ is the inversion efficiency (set to 0.85 here), $f$ is the tissue perfusion (i.e., cerebral blood flow; $\mathrm{CBF}$ ), $T_{1 a}$ is the T1 of arterial blood (set to $1.65 \mathrm{~s}$ here), $\tau$ is the label duration and $w$ is the post-label delay [15].

\section{Analysis}

The results were analyzed qualitatively by visual inspection of parameter maps, and quantitatively using homogeneity analysis for the $M_{0 a}$ maps and ROI analysis of perfusion estimates. The homogeneity was assessed by studying the dependence of $M_{0 a}$ values on the PVs of different tissue types. A higher homogeneity would yield more consistent $M_{0 a}$ values across different tissue mixtures. The relative range of values across different PV intervals $(0-10 \%, 10-20 \%, \ldots)$ were calculated as the difference between the maximum and minimum $M_{0 a}$ value (averaged across subjects), normalized by the mean value across all intervals. The homogeneity was further assessed with the so-called normalized absolute average deviation (UNAAD) score [16], calculated as $U N A A D=100\left(1-s_{n}\right)$ where $s_{n}$ is the normalized absolute deviation across the $M_{0 a}$ image. A higher value thus corresponds to a more uniform image. The score was calculated slice-by-slice and averaged across all slices. Subject-wise whole-brain GM and WM ROIs were obtained by thresholding $p_{i}$ values according to $p_{i}>75 \%$ and performing morphological closing (to approximately mimic manual delineation), and perfusion values in the ROIs were registered (mean and standard deviation). A signal-to-noise ratio (SNR) based thresholding was applied to remove voxels with unreliable perfusion estimates. The SNR of $\Delta M$ was calculated voxel-wise as the ratio of mean to standard deviation across the averages, and the threshold for inclusion was set to $\geq 0.1$. The Wilcoxon signed rank test was used to test for differences in perfusion values between the methods, with mean ROI values constituting paired data points for each subject $(n=7, \alpha=0.05)$. 


\section{Results}

Figure 1 displays parameter maps from all 13 slices in one subject for the proposed $M_{0 a}$ approach. Figure 2 constitutes a visual comparison between the conventional and the proposed method. The maps show $M_{0 a}$ and the corresponding maps included in the calculation (Eqs. 4 and 5), as well as resulting maps of perfusion and perfusion difference, in one slice of one subject. An example of the PV-based GM and WM ROIs is also displayed, together with population-wide whole-brain distributions for GM and WM perfusion. Visual comparison of $M_{0 a}$ and perfusion is also demonstrated for all 7 subjects in Figure 3.

The proposed method showed an overall higher $M_{0 a}$ homogeneity in all subjects, indicating a reduced bias compared with the conventional approach. Figure 4 shows the consistency of $M_{0 a}$ values across variable amounts of CSF, GM and WM. The proposed method had a higher consistency in all cases, and the corresponding relative ranges were reduced from $32.0 \%$ to $20.6 \%$ in CSF, from $16.0 \%$ to $5.5 \%$ in GM, and from $22.0 \%$ to $2.8 \%$ in WM. The proposed method had an average UNAAD score of $89.4 \pm 0.2$ (mean \pm standard error of mean; SEM), compared to the conventional method with a score of $84.4 \pm 1.6$. Supporting Table S1 includes the total homogeneity results in all subjects.

Results of the ROI analysis are compiled in Supporting Table S2, including mean, variability and differences in perfusion values across all subjects, for both ROIs and both methods. Mean GM perfusion over all subjects was (mean $\pm \mathrm{SEM}$ ) $61.2 \pm 1.7 \mathrm{ml} / 100 \mathrm{~g} / \mathrm{min}$ for the proposed method, and $56.3 \pm 1.6 \mathrm{ml} / 100 \mathrm{~g} / \mathrm{min}$ for the conventional method ( $8.7 \%$ difference). The corresponding WM values were $20.8 \pm 0.8 \mathrm{ml} / 100 \mathrm{~g} / \mathrm{min}$ for the proposed method and $22.0 \pm 0.9 \mathrm{ml} / 100 \mathrm{~g} / \mathrm{min}$ for the conventional method $(-5.4 \%$ difference).

The data and outcome of the statistical analysis are displayed in Figure 5. The proposed method yielded significantly higher perfusion values in GM and significantly lower perfusion values in WM, compared to the conventional method.

\section{Discussion}

The SR-based FSM produced generally consistent and realistic PV estimates (Fig. 1). The implemented calibration sequence was very fast $(18 \mathrm{~s})$, and can therefore be added to any protocol with minimal impact on total scan time. The proposed method produced $M_{0 a}$ maps with a high homogeneity throughout the brain (Fig. 1). The consistency analysis (Fig. 4) as well as the spatial homogeneity score confirmed the improved homogeneity and reduced tissue volume bias of the proposed method. However, the consistency was not perfect, and residual bias might still be an issue with the proposed method (especially in CSF contaminated voxels). This could be due to errors in PV estimates or incorrect literature values. Note that effects of B1 ${ }^{-}$ and $\mathrm{T}^{(*)}$ contrast are included in the $M_{0 a}$ maps, which is a desired feature since these effects will then be corrected for in the perfusion maps [7,8]. As such, the $M_{0 a}$ maps are not expected to be completely homogenous, and strictly speaking, 'calibration map' would be a more appropriate term than 'equilibrium magnetization of arterial blood' (since the latter is a global factor). Results from 7 healthy volunteers verified that the novel method consistently produced homogenous $M_{0 a}$ maps, which corresponds to a more accurate calibration (Fig. 3) $[8,10]$.

The statistical analysis showed that the differences in both GM and WM CBF values were statistically significant. The absolute differences between the methods varies spatially, as it depends on the local tissue composition and CSF PV effects. The largest difference between the two methods was found in GM, whereas WM perfusion values were slightly lower using the proposed method, compared to the conventional method. This difference between GM and WM is clearly seen in the difference maps (Fig. 3). The significantly higher GM perfusion estimates, yielded by the proposed method, are due to the use of a more accurate GM partition coefficient that is higher $(0.98 \mathrm{ml} / \mathrm{g})$ than the conventional whole-brain average $(0.9$ 
$\mathrm{ml} / \mathrm{g}$ ). The more homogenous $M_{0 a}$ acquired with the proposed method also implies that we have corrected for the inverse PD bias present in the conventional method [10]. Furthermore, we corrected for the fact that CSF is non-perfused, which means that we should obtain more accurate perfusion estimates in areas with CSF PV contamination, such as in cortical GM and deep GM structures. For example, in Figure 2, we clearly see higher perfusion values in the basal ganglia with the proposed method. The spatial and absolute differences between the two methods were consistent across all subjects (Fig. 3 and Supporting Table S2).

The proposed $M_{0 a}$ method corrects $M_{0}$ to only include the magnetization of perfused tissue, and calculates a tissue-weighted brain-blood partition coefficient map. A more subtle difference between the proposed and the conventional approach is how the $M_{0}$ of the voxel is measured. The conventional approach normally uses a single PD image with long repetition time, whereas, in the present work, $M_{0}$ was estimated from the fit of the SR signal equation to 9 images.

In Figure 1 we observe the common EPI susceptibility-induced signal loss around the paranasal sinuses. The FSM analysis is insensitive to this effect so it does not propagate to the $\lambda_{w}$ map, and the signal loss is therefore preserved in the $M_{0 a}$ map. As in the conventional method, this is desired since it effectively corrects for the corresponding loss in perfusion signal.

\section{Similar work}

With a similar objective, Dai et al. suggested to acquire a uniform calibration map using a multiple inversion recovery sequence [10]. The study showed that, the uniform calibration map produced perfusion values in better agreement with an approach in which the image was binary segmented into GM and WM and appropriate partition coefficients were used in the respective ROIs, compared to the conventional approach. As with our approach, a non-standard sequence was required. An advantage of the method of Dai et al. is that $M_{0}$ and $\lambda$ do not need to be mapped [10], while an advantage of our approach is that we also obtain FSM-based PV values. These native space tissue volume estimates can be used for generating ROIs, or for more advanced post-processing procedures such as partial volume correction (PVC) [17,18]. The use of PVC in ASL has gained increased interest in recent years, and the use of FSM-enabled sequences is expected to increase, which strengthens the importance of this novel $M_{0 a}$ method.

Cavusoglu et al. [7] and Chen et al. [19] previously reported on the dependence of perfusion estimates on the employed $M_{0 a}$ method by comparing several different approaches, although they did not assess the method proposed in this work. Both of these studies reported on substantial differences in perfusion values between various $M_{0 a}$ methods.

Petersen et al. suggested using $M_{0}$ or $T_{1}$ maps for estimating voxel-wise $\lambda$ values in model-free ASL [8]. This was achieved by binary segmentation of the brain into GM and WM, assignment of literature $\lambda_{i}$ values to the corresponding mean $M_{0}$ or $T_{1}$ values in GM/WM, and then calculation of the $\lambda$ map by linear interand extrapolation. Although this approach yielded a good approximation of the $\lambda$ map and homogenous $M_{0 a}$ maps, it assumes that the voxel $M_{0}$ or $T_{1}$ varies linearly with the tissue composition, and it does not accurately account for non-perfused tissue. With the same ASL data (QUASAR), it is possible to instead apply FSM and our proposed $M_{0 a}$ calculation [20,21].

\section{Challenges and limitations}

Some challenges and limitations of the work should also be noted. First, no correction for differences in T2* between tissue and blood was applied. This is a delicate correction which ideally should include tissue T2* mapping and corresponding appropriate voxel-wise correction, although literature tissue T2* values have commonly been used. Such a correction would not change the relative differences between the proposed and the conventional method, and this limitation does therefore not affect the conclusions of this work. 
Second, the calibration should ideally be independent of literature $\lambda$ values, and partition coefficient mapping would be favorable [13]. However, this is a challenging problem in itself, and no standard MRI method has been established. Partition coefficient values as well as PV estimation might also be unreliable in patients with pathological tissue changes.

Third, the method relies on the availability of PV estimates. The FSM approach has been shown to produce reliable PV estimates with high repeatability and robustness to noise [14,18,22]. Furthermore, FSM can be implemented to yield PV estimates in the native space of ASL data (as in this work, and Refs. $[17,18]$ ). The respective accuracies of $M_{0}, T_{1}$ and PV estimates depend on the SNR, and the quality of the results should always be assessed in relation to the specific experimental conditions (for example, if a higher spatial resolution is employed). PV contamination from other tissue types or pathologies could also introduce errors in the PV values and, subsequently, the perfusion values. If FSM is unavailable, it may be feasible to use segmentation of the ASL data (e.g., $M_{0}$ image), separate T1- or T2-weighted images using the same readout, or a high-resolution scan. Alternatively, to reduce the bias in GM perfusion, $\lambda_{G M}$ may be used instead of $\bar{\lambda}$ if the WM perfusion is dismissed.

Fourth, WM perfusion values are generally unreliable for standard ASL acquisitions. This is mainly due to the low SNR, and only a fraction of the WM voxels normally show significant perfusion signal [23-25]. An SNR based thresholding was applied in this work to reduce the impact of unreliable voxels, especially WM voxels. Still, the WM perfusion values are reported primarily to facilitate the comparison between the $M_{0 a}$ methods, and they should not be over-interpreted to represent reliable perfusion values in absolute terms.

Lastly, the use of the brain-blood partition coefficient is not entirely straightforward in basic ASL modelling. This is mainly because the standard simplified ASL models assume that the labeled blood water instantly exchanges with tissue upon arrival to the capillaries, so that $\lambda$ represents the distribution volume. However, this assumption is not completely accurate, and models including water exchange between blood and tissue [26-28] or model-free approaches [6], can be used for a more complete analysis and use of $\lambda$. In contrast to the simplified model, the more advanced exchange models do not assume that the blood and tissue water is in equilibrium, and the application of $\lambda$ is thus different. However, the proposed method for improved calculation of $M_{0 t}$ and $\lambda_{w}$ would still be valid for more advanced ASL models.

\section{Conclusions}

The equilibrium magnetization of arterial blood water, $M_{0 a}$, is the central calibration factor in ASL, and proper $M_{0 a}$ calculation is essential to obtain accurate perfusion estimates. We have therefore proposed an improved method which exploits PV estimates to obtain an $M_{0 a}$ map with appropriate adjustment for the distribution volume of labeled blood water. Compared to the conventional approach, the proposed method produced more homogenous $M_{0 a}$ maps and significantly different perfusion values. Although the conventional $M_{0 a}$ method should still be regarded as the recommended approach for clinical routine and basic applications [15], the new $M_{0 a}$ approach, reported in this study, is likely to improve quantitative perfusion estimation with ASL, and may therefore be of considerable value in more specialized perfusion imaging studies.

\section{Acknowledgements}

This study was supported by the Swedish Research Council (grant nos. 2011-2971 and 2017-00995). The authors would also like to thank the European COST Action BM1103, 'Arterial Spin Labelling Initiative in Dementia' (AID), for supporting the collaboration enabling this work. 


\section{References}

1. Haller S, Zaharchuk G, Thomas DL, Lovblad KO, Barkhof F, Golay X. Arterial spin labeling perfusion of the brain: Emerging clinical applications. Radiology 2016;281:337-356.

2. $\quad$ Detre JA, Leigh JS, Williams DS, Koretsky AP. Perfusion imaging. Magn Reson Med 1992;23:3745.

3. Williams DS, Detre JA, Leigh JS, Koretsky AP. Magnetic resonance imaging of perfusion using spin inversion of arterial water. Proc Natl Acad Sci U S A 1992;89:212-216.

4. Wong EC, Buxton RB, Frank LR. Implementation of quantitative perfusion imaging techniques for functional brain mapping using pulsed arterial spin labeling. NMR Biomed 1997;10:237-249.

5. Chalela JA, Alsop DC, Gonzalez-Atavales JB, Maldjian JA, Kasner SE, Detre JA. Magnetic resonance perfusion imaging in acute ischemic stroke using continuous arterial spin labeling. Stroke 2000;31:680-687.

6. Petersen ET, Lim T, Golay X. Model-free arterial spin labeling quantification approach for perfusion MRI. Magn Reson Med 2006;55:219-232.

7. Cavusoglu M, Pfeuffer J, Ugurbil K, Uludag K. Comparison of pulsed arterial spin labeling encoding schemes and absolute perfusion quantification. Magn Reson Imaging 2009;27:10391045.

8. Petersen ET, Zimine I, Ho Y-CL, Golay X. An improved QUASAR sequence for user-independent quantitative and reproducible perfusion measurements. In Proceedings of the 15th Annual Meeting of ISMRM, Berlin, Germany, 2007. p. 376.

9. Herscovitch P, Raichle ME. What is the correct value for the brain-blood partition coefficient for water? J Cereb Blood Flow Metab 1985;5:65-69.

10. Dai W, Robson PM, Shankaranarayanan A, Alsop DC. Sensitivity calibration with a uniform magnetization image to improve arterial spin labeling perfusion quantification. Magn Reson Med 2011;66:1590-1600.

11. Lassen NA, Perl W. Tracer kinetic methods in medical physiology. New York: Raven Press; 1979.

12. Buxton RB, Frank LR, Wong EC, Siewert B, Warach S, Edelman RR. A general kinetic model for quantitative perfusion imaging with arterial spin labeling. Magn Reson Med 1998;40:383-396.

13. Roberts DA, Rizi R, Lenkinski RE, Leigh JS, Jr. Magnetic resonance imaging of the brain: blood partition coefficient for water: application to spin-tagging measurement of perfusion. J Magn Reson Imaging 1996;6:363-366.

14. Shin W, Geng X, Gu H, Zhan W, Zou Q, Yang Y. Automated brain tissue segmentation based on fractional signal mapping from inversion recovery Look-Locker acquisition. Neuroimage 2010;52:1347-1354.

15. Alsop DC, Detre JA, Golay X, Gunther M, Hendrikse J, Hernandez-Garcia L, Lu H, MacIntosh BJ, Parkes LM, Smits M, van Osch MJ, Wang DJ, Wong EC, Zaharchuk G. Recommended implementation of arterial spin-labeled perfusion MRI for clinical applications: A consensus of the ISMRM perfusion study group and the European consortium for ASL in dementia. Magn Reson Med 2015;73:102-116.

16. Goerner FL, Duong T, Stafford RJ, Clarke GD. A comparison of five standard methods for evaluating image intensity uniformity in partially parallel imaging MRI. Med Phys 2013;40:082302.

17. Petr J, Schramm G, Hofheinz F, Langner J, van den Hoff J. Partial volume correction in arterial spin labeling using a Look-Locker sequence. Magn Reson Med 2013;70:1535-1543.

18. Ahlgren A, Wirestam R, Petersen ET, Stahlberg F, Knutsson L. Partial volume correction of brain perfusion estimates using the inherent signal data of time-resolved arterial spin labeling. NMR Biomed 2014;27:1112-1122.

19. Chen Y, Wang Z, Detre JA. Impact of equilibrium magnetization of blood on ASL quantification. In Proceedings of the 19th Annual Meeting of ISMRM, Montréal, Canada, 2011. p. 300. 
20. Ahlgren A, Wirestam R, Ståhlberg F, Knutsson L. Using fractional segmentation for estimation of the equilibrium magnetization of arterial blood in model-free arterial spin labeling. In Proceedings of the 28th Annual Scientific Meeting of ESMRMB, Leipzig, Germany, 2011. p. 18.

21. Ahlgren A, Wirestam R, Petersen ET, Stahlberg F, Knutsson L. Perfusion quantification by modelfree arterial spin labeling using nonlinear stochastic regularization deconvolution. Magn Reson Med 2013;70:1470-1480.

22. Ahlgren A, Wirestam R, Stahlberg F, Knutsson L. Automatic brain segmentation using fractional signal modeling of a multiple flip angle, spoiled gradient-recalled echo acquisition. Magn Reson Mater Phy 2014;27:551-565.

23. van Osch MJ, Teeuwisse WM, van Walderveen MA, Hendrikse J, Kies DA, van Buchem MA. Can arterial spin labeling detect white matter perfusion signal? Magn Reson Med 2009;62:165-173.

24. Wu WC, Lin SC, Wang DJ, Chen KL, Li YD. Measurement of cerebral white matter perfusion using pseudocontinuous arterial spin labeling 3T magnetic resonance imaging - an experimental and theoretical investigation of feasibility. PLoS One 2013;8:e82679.

25. Skurdal MJ, Bjornerud A, van Osch MJ, Nordhoy W, Lagopoulos J, Groote IR. Voxel-wise perfusion assessment in cerebral white matter with PCASL at 3T; Is it possible and how long does it take? PLoS One 2015;10:e0135596.

26. St. Lawrence KS, Frank JA, McLaughlin AC. Effect of restricted water exchange on cerebral blood flow values calculated with arterial spin tagging: A theoretical investigation. Magn Reson Med 2000;44:440-449.

27. Zhou J, Wilson DA, Ulatowski JA, Traystman RJ, van Zijl PC. Two-compartment exchange model for perfusion quantification using arterial spin tagging. J Cereb Blood Flow Metab 2001;21:440455.

28. Parkes LM, Tofts PS. Improved accuracy of human cerebral blood perfusion measurements using arterial spin labeling: accounting for capillary water permeability. Magn Reson Med 2002;48:2741. 


\section{Figures and tables}

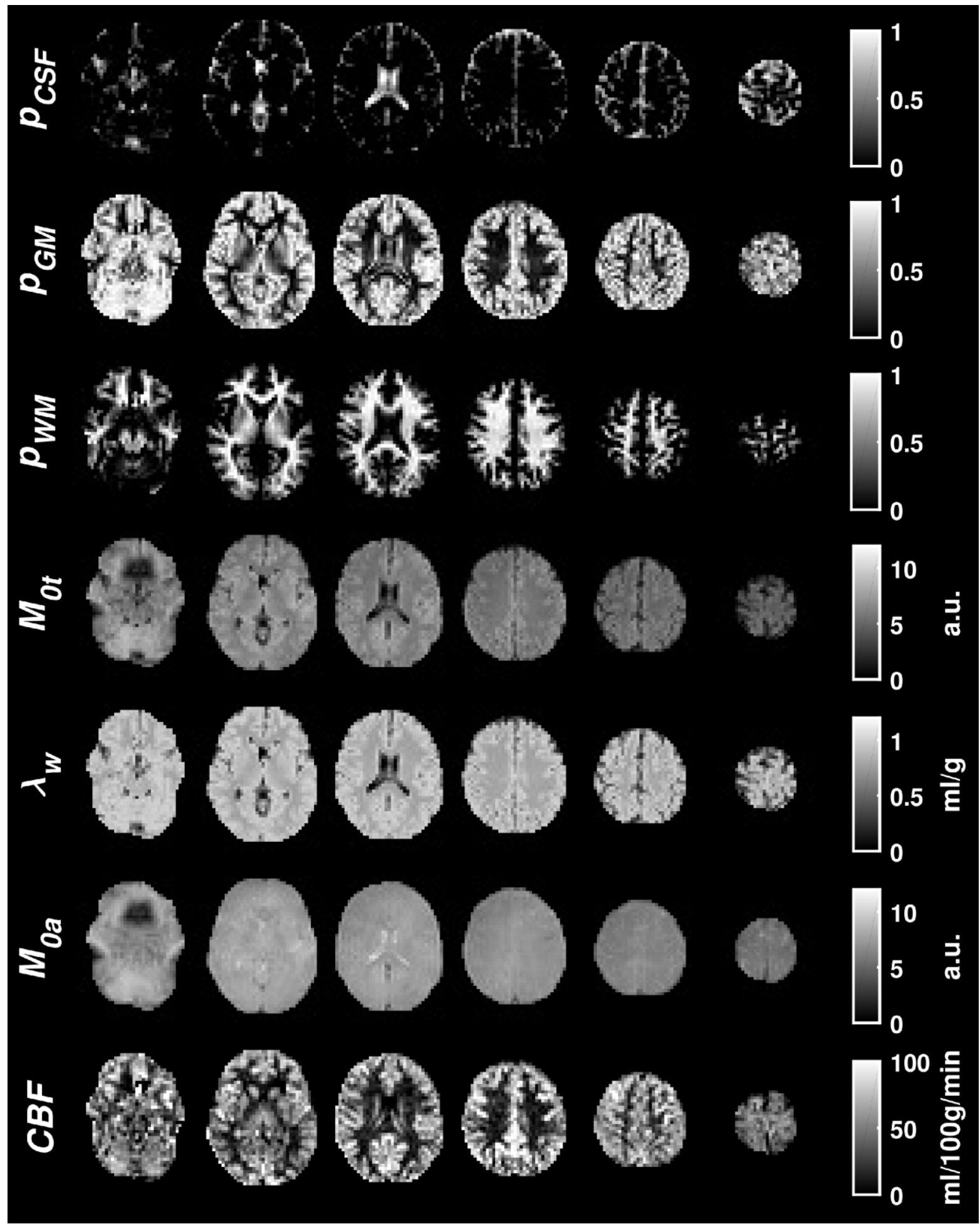

Figure 1. Parameter maps from 6 slices in one subject obtained using the proposed method. The top three rows show PV estimates in CSF, GM and WM. The subsequent rows display the equilibrium magnetization of perfused tissue $\left(M_{0 t}\right)$, the tissue-weighted brain-blood partition coefficient $\left(\lambda_{w}\right)$, the equilibrium magnetization of arterial blood $\left(M_{0 a}\right)$, and the tissue perfusion $(C B F)$. 


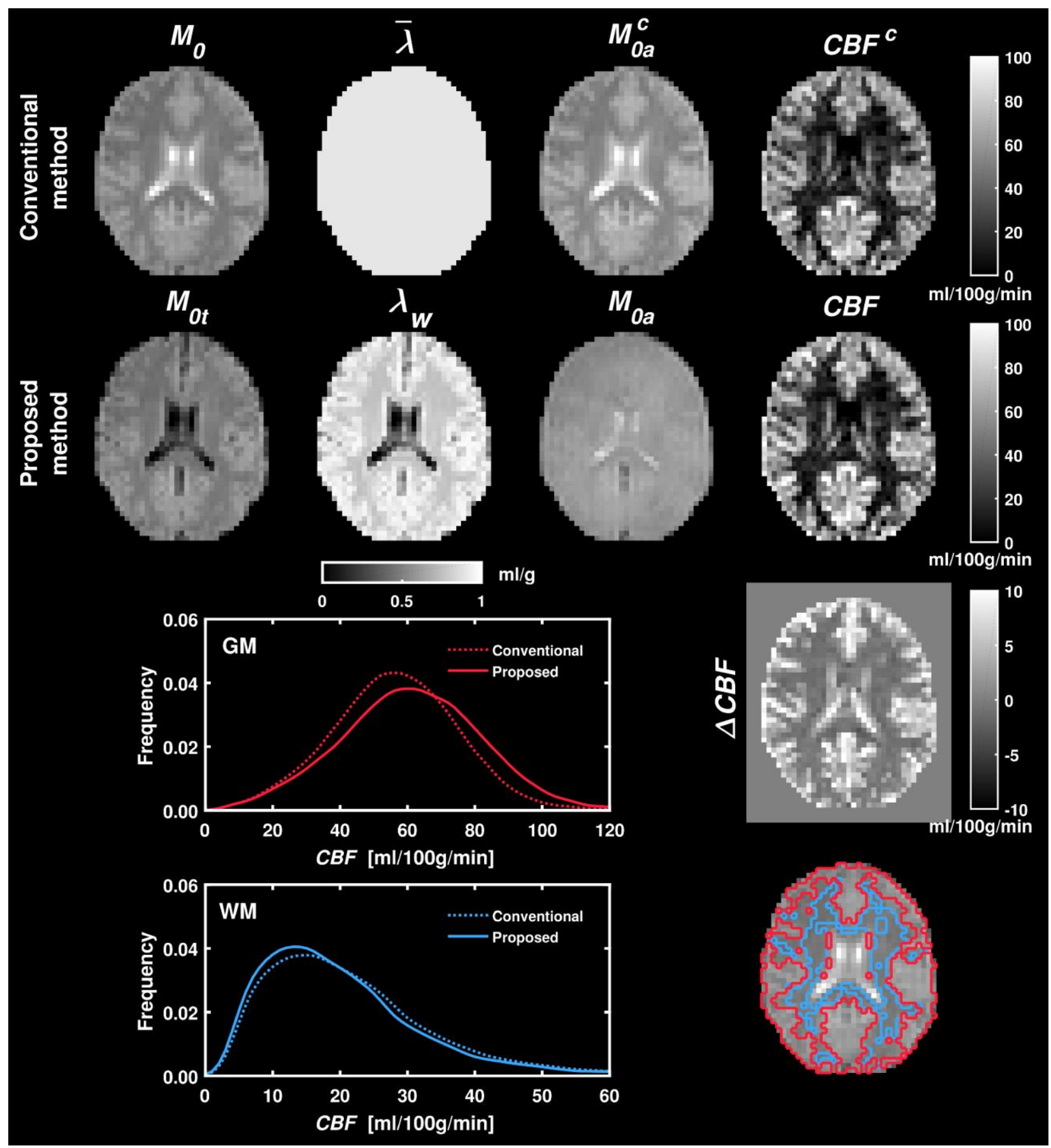

Figure 2. Comparison between the conventional and the proposed method, in one slice of one subject. The top row shows the maps included in the conventional method (Eq. 5) and the resulting perfusion map. The second row shows the corresponding maps for the proposed method (Eq. 4). A perfusion difference map is displayed below the perfusion images $\left(\triangle C B F=C B F-C B F^{c}\right)$. In the bottom right, an example of the PVbased GM (red) and WM (blue) ROIs is displayed. The two graphs show whole-brain GM and WM perfusion distributions (kernel smoothing) after application of the SNR-based thresholding, over all 7 subjects for the conventional (dashed lines) and the proposed (solid lines) method, in corresponding colors. 


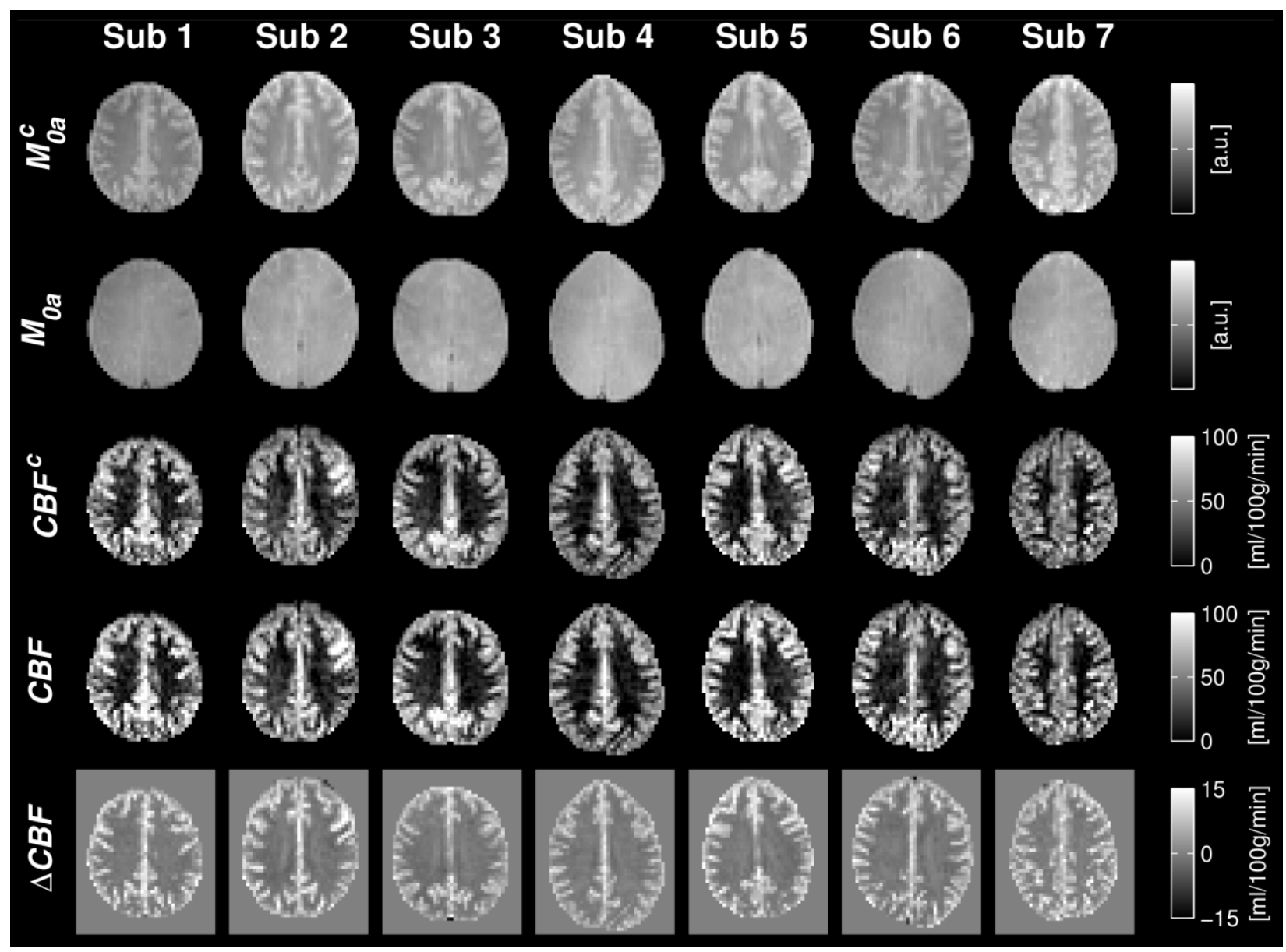

Figure 3. Comparison of both $M_{0 a}$ methods in all 7 subjects. The top two rows display the calibration maps with the conventional and the proposed method. The third and fourth rows display the perfusion maps using the conventional and proposed method, respectively, and the bottom row displays the perfusion difference maps $\left(\triangle C B F=C B F-C B F^{c}\right)$. 
a)

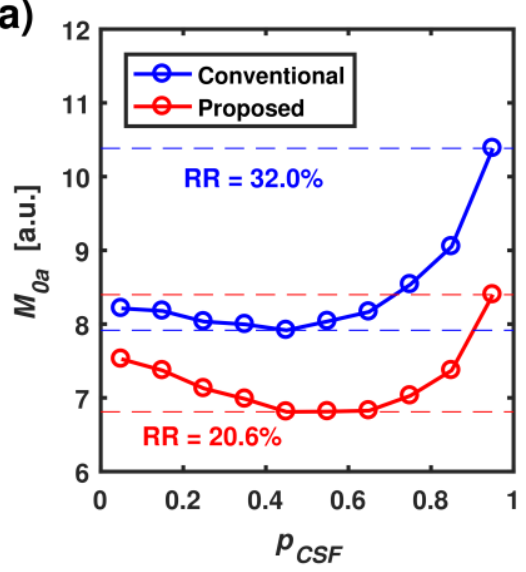

b)

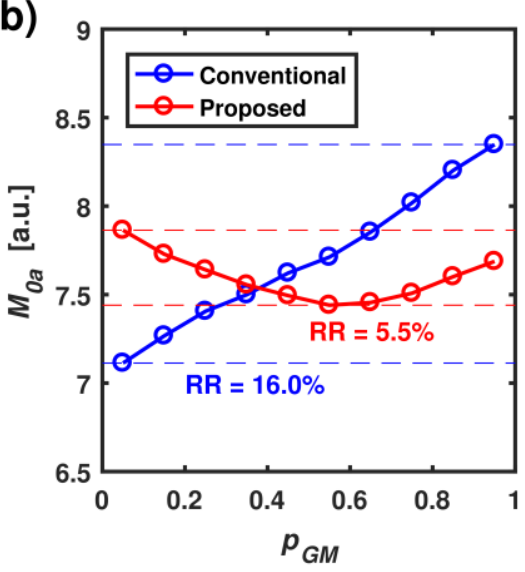

c)

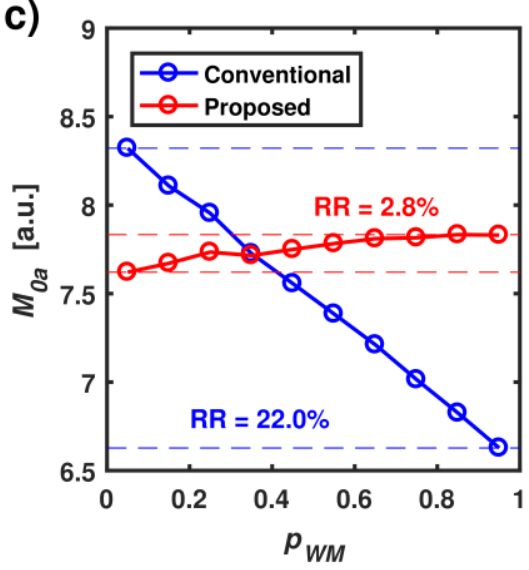

Figure 4. Consistency of $M_{0 a}$ values (averaged over all subjects) across different PV intervals of (a) CSF, (b) GM, and (c) WM. Circles connected with solid lines correspond to $M_{0 a}$ values for the conventional method (blue) and the proposed method (red). The dashed lines show the maximum and minimum value across all PV intervals, in corresponding colors. The relative range (RR) of values across all PV intervals is also displayed in corresponding colors. The proposed method had a reduced dependence on PVs across all tissue types. 
a)

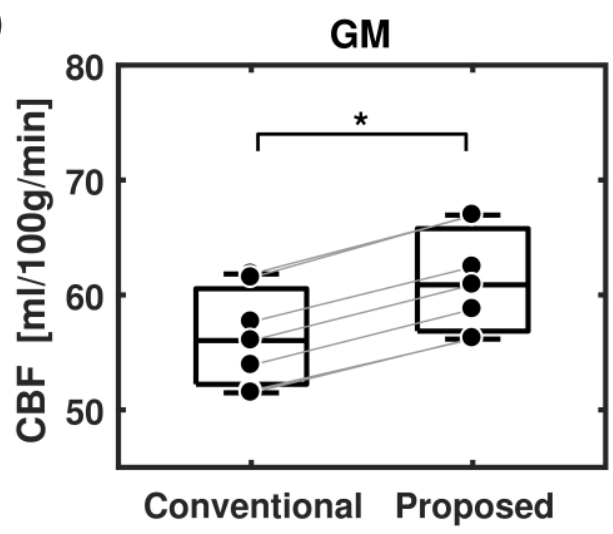

b)

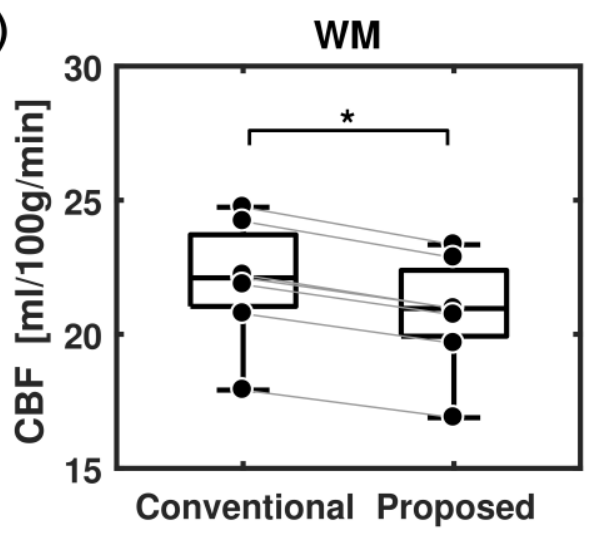

Figure 5. Differences between the conventional and the proposed method, visualized with box-and-whisker plots, including paired data points (corresponding to the 7 subjects). The pairs are indicated by interconnecting gray lines. Significant differences (asterisk) in perfusion estimates were found in both (a) GM and (b) WM, using a paired test (Wilcoxon signed rank test, $\alpha=0.05$ ). 
Supporting Table S1. $M_{0 a}$ homogeneity analysis using the normalized absolute average deviation (UNAAD) score [Goerner et al. Med Phys 2013;40:082302]. A higher value corresponds to a more uniform image.

\begin{tabular}{|c|l|l|}
\hline Subject & $\begin{array}{c}\text { UNAAD } \\
\text { conventional }\end{array}$ & $\begin{array}{c}\text { UNAAD } \\
\text { proposed }\end{array}$ \\
\hline 1 & 86.8 & 89.9 \\
\hline 2 & 74.7 & 89.8 \\
\hline 3 & 85.9 & 88.8 \\
\hline 4 & 86.9 & 89.7 \\
\hline 5 & 84.7 & 89.0 \\
\hline 6 & 86.1 & 89.7 \\
\hline 7 & 85.5 & 89.2 \\
\hline Mean $\mathbf{\text { SEM }}$ & $\mathbf{8 4 . 4} \pm \mathbf{1 . 6}$ & $\mathbf{8 9 . 4} \pm \mathbf{0 . 2}$ \\
\hline
\end{tabular}

Supporting Table S2. Results of the ROI analysis. Mean and standard deviation (SD) are reported for each subject, for both ROIs and both methods. Differences are reported in absolute and relative values. The bottom row shows the mean and standard error of mean (SEM) across all subjects (or mean absolute and relative values for the differences). All perfusion values are in $\mathrm{ml} / 100 \mathrm{~g} / \mathrm{min}$.

\begin{tabular}{|c|l|l|l|l|l|l|}
\hline Subject & $\begin{array}{c}\text { GM perfusion } \\
\text { conventional } \\
{[\text { mean } \pm \text { SD] }}\end{array}$ & $\begin{array}{c}\text { GM perfusion } \\
\text { proposed } \\
{[\text { mean } \pm \text { SD] }}\end{array}$ & $\begin{array}{c}\text { GM perfusion } \\
\text { difference }\end{array}$ & $\begin{array}{c}\text { WM perfusion } \\
\text { conventional } \\
{[\text { mean } \pm \text { SD] }}\end{array}$ & $\begin{array}{c}\text { WM perfusion } \\
\text { proposed } \\
{[\text { mean } \pm \text { SD] }}\end{array}$ & $\begin{array}{c}\text { WM perfusion } \\
\text { difference }\end{array}$ \\
\hline 1 & $57.7 \pm 18.7$ & $62.4 \pm 21.1$ & $4.8(8.3 \%)$ & $22.1 \pm 16.2$ & $21.0 \pm 16.0$ & $-1.1(-5.1 \%)$ \\
\hline 2 & $53.9 \pm 18.0$ & $58.8 \pm 20.4$ & $4.9(9.0 \%)$ & $24.7 \pm 14.0$ & $23.3 \pm 13.9$ & $-1.4(-5.7 \%)$ \\
\hline 3 & $61.8 \pm 18.6$ & $66.9 \pm 20.9$ & $5.1(8.2 \%)$ & $24.2 \pm 15.5$ & $22.9 \pm 15.2$ & $-1.4(-5.6 \%)$ \\
\hline 4 & $56.0 \pm 17.4$ & $60.9 \pm 19.8$ & $4.9(8.7 \%)$ & $20.8 \pm 12.5$ & $19.7 \pm 12.6$ & $-1.1(-5.3 \%)$ \\
\hline 5 & $61.5 \pm 19.3$ & $66.9 \pm 22.0$ & $5.4(8.9 \%)$ & $22.2 \pm 16.4$ & $21.0 \pm 15.7$ & $-1.3(-5.6 \%)$ \\
\hline 6 & $51.7 \pm 18.1$ & $56.2 \pm 20.4$ & $4.5(8.7 \%)$ & $21.9 \pm 17.7$ & $20.7 \pm 17.2$ & $-1.1(-5.2 \%)$ \\
\hline 7 & $51.5 \pm 18.6$ & $56.2 \pm 21.2$ & $4.7(9.2 \%)$ & $17.9 \pm 10.8$ & $16.9 \pm 10.6$ & $-1.0(-5.7 \%)$ \\
\hline Mean \pm SEM & $\mathbf{5 6 . 3} \pm \mathbf{1 . 6}$ & $\mathbf{6 1 . 2} \pm \mathbf{1 . 7}$ & $\mathbf{4 . 9}(\mathbf{8 . 7 \%})$ & $\mathbf{2 2 . 0} \pm \mathbf{0 . 9}$ & $\mathbf{2 0 . 8} \pm \mathbf{0 . 8}$ & $\mathbf{- 1 . 2 ( - 5 . 4 \% )}$ \\
\hline
\end{tabular}

